\title{
INTERNET COMO FUENTE DE INFORMACIÓN PARA LA VIDA COTIDIANA DE LOS JÓVENES ESPAÑOLES
}

\author{
Jordi Sánchez-Navarro y Daniel Aranda
}
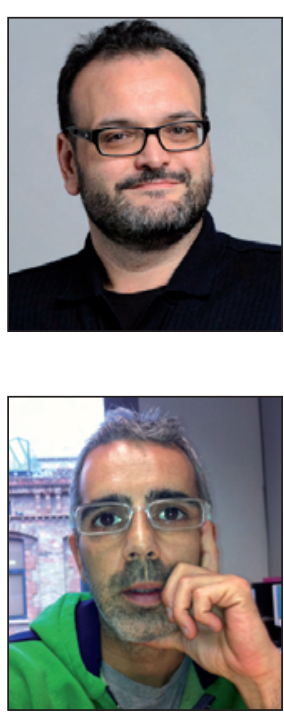

Jordi Sánchez-Navarro es doctor en comunicación audiovisual por la Universitat Ramon Llull. Profesor de los estudios de ciencias de la información y la comunicación de la Universitat Oberta de Catalunya (UOC) y miembro del grupo de investigación Communication and New Media del Internet Interdisciplinary Institute (IN3). Forma parte del equipo de investigadores del proyecto Prácticas creativas y participación en los nuevos medios, financiado por el Plan Nacional de I+D+i del Ministerio de Ciencia e Innovación (2010-2013).

Universitat Oberta de Catalunya Rambla del Poblenou, 156. 08018 Barcelona jsancheznav@uoc.edu

Daniel Aranda es doctor en comunicación audiovisual por la Universitat Ramon Llull. Profesor de los estudios de ciencias de la información y la comunicación de la Universitat Oberta de Catalunya (UOC) y miembro del grupo de investigación Communication and New Media del Internet Interdisciplinary Institute (IN3). Forma parte del equipo de investigadores del proyecto El uso de las TIC y la brecha digital entre adultos y adolescentes, financiado por el Plan Nacional de I+D+i del Ministerio de Ciencia e Innovación (2009-2012).

Universitat Oberta de Catalunya Rambla del Poblenou, 156. 08018 Barcelona darandaj@uoc.edu

\section{Resumen}

Se muestran y discuten datos cuantitativos obtenidos mediante una encuesta dirigida a población española de entre 12 y 18 años, así como observaciones cualitativas de diversos grupos de discusión, sobre el uso de internet como medio de obtención de información útil para la vida cotidiana, que se desarrolla en dos ámbitos: el ocio y la educación formal. Los datos nos muestran una juventud que utiliza internet en entornos informales y privados y en menor medida en espacios educativos formales. Para este grupo de población, la Red se utiliza y merece ser explorada en la medida en que la información que se encuentra es útil para la sociabilidad y el ocio, mientras que sus beneficios en los estudios se dan por supuestos. Por último se destaca que los jóvenes desarrollan sus propias habilidades para la evaluación de la información.

\section{Palabras clave}

Internet, Sociabilidad, Vida cotidiana, Juventud, Cultura participativa.

\section{Title: Internet as information source for the everyday life of Spanish young people}

\begin{abstract}
This article reports and discusses quantitative data obtained from a survey of the Spanish population ages 12 to 18 , as well as qualitative observations obtained from various discussion groups on the use of the internet as a means of obtaining useful information for everyday life, particularly in two spheres: leisure and formal education. The data show that young people use the internet mainly in informal and private spaces and, to a lesser extent, in formal educational environments. For this population group, the internet is used and is worth exploring to the extent that the information found is useful for social and leisure purposes, while educational benefits are taken for granted. Lastly, we point out that the young develop their own skills for evaluating information.
\end{abstract}

\section{Keywords}

Internet, Sociability, Everyday life, Youth, Participatory culture.

Sánchez-Navarro, Jordi; Aranda, Daniel. "Internet como fuente de información para la vida cotidiana de los jóvenes españoles". El profesional de la información, 2011, enero-febrero, v. 20, n. 1, pp. 32-37.

DOI: 10.3145/epi.2011.ene.04 


\section{Introducción}

Los jóvenes se han convertido en un objeto de estudio privilegiado en el campo de la reflexión sobre el impacto social y cultural del uso y consumo de las tecnologías de la información y la comunicación (TIC). Son la generación digital, la vanguardia que representa el futuro, pero también el sector de población más vulnerable a los riesgos que encierran estas tecnologías. Los niños y los adolescentes suelen ser considerados un objeto singular de estudio, una categoría homogénea con la capacidad de apropiarse de las tecnologías digitales de maneras diferentes y más innovadoras que sus mayores (Livingstone, 2003; 2007; 2008; Buckingham, 2008). Las metáforas implícitas en estos enfoques han dado lugar a categorías teóricas como las de nativo digital (Prensky, 2001), generación digital (Rubio-Gil, 2010) o generación interactiva (Bringué; Sádaba, 2008). La otra cara es la que representa el joven "necesitado", que carece de las habilidades requeridas para aprovechar al máximo esas tecnologías (Selwyn, 2003).

Las TIC afectan a los jóvenes en su vida cotidiana, entendida, tal y como hacen los estudios culturales, como algo articulado por la cultura y con ella. Estos estudios describen cómo las estructuras y fuerzas particulares que organizan sus vidas cotidianas de maneras contradictorias conceden o niegan capacidades a las personas, que desarrollan prácticas culturales para definir contextos y relacionarse con ellos (De-Certeau, 1984; Geertz, 1995; Orozco, 1996; Lull, 1997; Goffman, 1994; Storey, 1999; Highmore, 2001; Grossberg, 2009).

Los jóvenes realizan prácticas culturales en todos los ámbitos de socialización, aunque aquí destacaremos dos: el ocio y la educación formal. En relación con estas prácticas la familia, como agente de socialización primaria, tiene un papel clave en la introducción de los más jóvenes en las tecnologías. Sin embargo, de todos los papeles que cabe atribuirle, parece que el que adopta con mayor naturalidad es el de ente regulador, puesto que en la mayoría de ocasiones los términos de las discusiones familiares se reducen a la cantidad de horas y la frecuencia que los adolescentes pasan en contacto con los medios de comunicación y la tecnología (Dickinson et al., 2001; Livingstone; Helsper, 2007; Hagen, 2007). A menudo este debate se reduce a la dicotomía "instrumento de placer/instrumento de trabajo", o más en abstracto a la dicotomía "trabajo/juego" (McMillan; Morrison, 2006).

Estas dicotomías no parecen ser instrumentos teóricos adecuados para explicar el vínculo de los jóvenes con internet, dado que las acciones que estos realizan en la Red repercuten de manera significativa en su dinámica de obtención y desarrollo de competencias desde el punto de vista social, cultural y educativo, es decir, en la manera que tienen de comunicarse, consumir, trabajar, estudiar, colaborar y resolver problemas (Aranda; Sánchez-Navarro; Tabernero, 2009; Espinar-Ruiz; González-Río, 2009; Gordo-López, 2009; Rubio-Gil, 2009; 2010; Bernete, 2010). En este contexto el conocimiento, ya sea cultural, profesional o técnico, se comparte de manera informal y merece el reconocimiento de todos los implicados (Jenkins et al., 2008). La juventud adquiere así capital-red social, o conocimiento asociado a la contribución propia y de los demás a la comunidad, al compartir sus experiencias y opiniones en espacios emergentes de apoyo, sociabilidad y reconocimiento, sustentados por relaciones de amistad y/o interés, en los que se produce un aprendizaje colaborativo (Rheingold, 2002; Ito et al., 2009; Gee, 2004).

En este entorno es relevante tener en cuenta los usos, percepciones y actitudes de los jóvenes sobre la información disponible en internet y su búsqueda, consumo y reutilización. Este artículo es una aproximación descriptiva a esas cuestiones a partir de los datos obtenidos en el marco de una investigación sobre las prácticas de ocio y sociabilidad de los jóvenes, así como las dinámicas de aprendizaje informal que se producen en su uso cotidiano de internet ${ }^{1}$.

Los adolescentes entienden internet como un espacio de ocio, separado de los contextos educativos formales

\section{Método}

Para la obtención de los datos cuantitativos se realizó una encuesta telefónica cuyo universo era la población de entre 12 y 18 años de España. Se generó una muestra teórica final de 2.054 consultas con un margen de error de $\pm 2,16 \%$ para $\mathrm{P}=\mathrm{Q}=50,0 \%$ y bajo el supuesto de máxima indeterminación. El número de consultas realizadas siguió una distribución proporcional a la población española tanto por sexo como por edad, así como por tamaño de municipio y por comunidades. El procedimiento de muestreo siguió una selección polietápica del entrevistado según este esquema:

- municipios, seleccionados de forma aleatoria proporcional para cada comunidad autónoma;

- hogares, mediante la selección aleatoria de números telefónicos;

- individuos, seleccionando a la persona del hogar de entre 12 y 18 años.

El trabajo de campo se realizó entre los días 16 de marzo y 1 de abril de 2009. El cuestionario constaba de un primer bloque sobre internet en general, en el que se preguntaba a los jóvenes sobre sus horarios y lugares de conexión, el tiempo dedicado, normas de control por parte de los padres, percepciones sobre la utilidad y usos efectivos, y un segundo bloque sobre el uso de servicios concretos: cuentas de mensajería instantánea, redes sociales, blogs y fotoblogs.

Los datos cualitativos se obtuvieron mediante la realización de una serie de grupos de discusión para estudiar en profundidad las opiniones de los jóvenes. Los grupos se reunieron en ocho centros de educación secundaria y cada uno de ellos constaba de ocho jóvenes de ambos sexos. Los centros estaban situados en ciudades de cuatro comunidades autónomas elegidas por el nivel de implantación y uso de internet según el Estudio General de Medios y el Instituto Nacional de Estadística. Las cuatro comunidades seleccionadas fueron Catalunya y Madrid (alta penetración) y Andalucía y Galicia (baja penetración). Dentro de las comunidades se seleccionaron las ciudades de Barcelona, Madrid, Tarifa y San- 
tiago de Compostela, y en cada una de ellas dos institutos del sistema público de educación. En cada instituto se realizaron dos grupos, uno con estudiantes de ESO (enseñanza secundaria obligatoria) y otro con estudiantes de bachillerato. En los grupos de enfoque se recogieron datos sobre los usos, percepciones y opiniones sobre tiempos de conexión, servicios (buscadores, blogs, redes sociales), restricciones y usos concretos (jugar, chatear, trabajar en grupo, aficiones o buscar y generar información sobre materias de interés personal como música, deporte y otras).

Los grupos de discusión se realizaron entre los meses de abril y mayo de 2009. El contenido de las discusiones de los grupos se registró y transcribió para ser codificado con el software para análisis cualitativo Atlas.Ti. Para citar los comentarios y opiniones de los participantes velando por su anonimato, en las páginas siguientes se atribuyen a la fuente sólo tres datos: el género (chico/chica), la etapa formativa (ESO/bachillerato) y la ciudad de residencia.

\section{Resultados y discusión}

\subsection{Internet como infraestructura básica.}

Un primer dato relevante es que la práctica totalidad de los adolescentes en España afirma haber conectado alguna vez con internet $(96,7 \%)$. Además la mayoría lo hace con regularidad (el 53\% como mínimo una hora diaria como media, y resulta también significativo que un $13,6 \%$ del total afirma estar casi siempre conectado). Cabe mencionar que si bien un 3,3\% no lo utiliza, este porcentaje aumenta hasta un $5,3 \%$ en las chicas de entre 12 y 15 años.

Internet es para los adolescentes un instrumento de uso básico, doméstico y diario que de hecho deriva de (y le da sentido a) la presencia de ordenadores en el hogar; por ejemplo:

- chico, bachillerato, Santiago de Compostela: "yo no le encuentro utilidad al ordenador sin internet";

- chico, bachillerato, Madrid: "yo reconozco que [lo uso] todos los días porque no tengo nada que hacer en mi casa; si no tuviera ordenador...".

Respecto al lugar, la frecuencia e intensidad de uso, así como el control parental efectivo sobre este uso, hay que destacar que la gran mayoría $(94,5 \%)$ conecta habitualmente en casa, y que un $59,2 \%$ afirma tener conexión en su propia habitación. La disponibilidad de internet en espacios privados o personales se incrementa con la edad (es más frecuente entre los que tienen entre 16 y 18 años que entre los que tienen entre 12 y 15 años). Lo mismo ocurre con el tiempo de dedicación, ligeramente superior entre los más mayores, y con la migración gradual del horario principal de uso, desde las tardes (caso mayoritario, pero más común entre los que tiene entre 12 y 15 años) hacia las noches o la conexión a cualquier hora.

Resultan interesantes los datos sobre la vía de introducción al uso de internet. El 53,6\% afirma haber aprendido a utilizar la Red de forma autodidacta, mientras que el $21,8 \%$ ha aprendido con ayuda de algún familiar. Cabe destacar que el peso de la familia como vía de aprendizaje del uso de internet se nota específicamente entre los más pequeños (de 12 a 15 años). En conjunto los datos revelan que la gran mayoría (un 79,3\%) aprende a utilizar internet en contextos informales, ya sea por su cuenta, con la familia o con los amigos y amigas (3,9\%), y por tanto no relacionados con la educación reglada (apenas un 19,9\% afirma haber aprendido en clase o en academias).

En los grupos de discusión se puso de manifiesto que los adolescentes entienden internet en primer lugar como un espacio de ocio, vinculado a los contextos educativos formales sólo cuando es estrictamente necesario. A las preguntas “¿lo utilizáis para estudiar o buscar información?”, “¿en el instituto os recomiendan páginas de internet?", los jóvenes responden:

- chica, ESO, Santiago de Compostela: "las [páginas] educativas no son muy interesantes";

- investigador: "¿por qué?";

- chica, ESO, Santiago de Compostela: "porque no aportan nada de ocio, que es lo que buscamos";

- investigador: "¿lo usas sólo para entretenerte?";

- chico, ESO, Santiago de Compostela: "hombre, es que estás para que te den educación en un sitio seis horas, ocho horas, y llegas a casa y te metes en internet supuestamente para desconectar".

Los jóvenes reconocen que les faltan competencias para valorar la veracidad o utilidad de la información que obtienen en internet

\subsection{Entretenimiento e información.}

Con respecto a los usos específicos de internet, el entretenimiento (pasar el rato, bajar música, películas o videojuegos) y la información, tanto sobre intereses generales (informarse sobre temas de interés) como estrictamente prácticos (buscar información sobre trabajos o deberes del colegio o del instituto), son los dos usos principales que la juventud hace de la Red, por delante de la comunicación (relacionarse con gente), o del uso de aplicaciones que implican participación directa, como distribuir fotos, vídeos y opiniones (figura 1).

El hecho de que tanto en las respuestas abiertas de la encuesta como en los comentarios obtenidos en los grupos de discusión, los jóvenes separen el consumo de televisión y

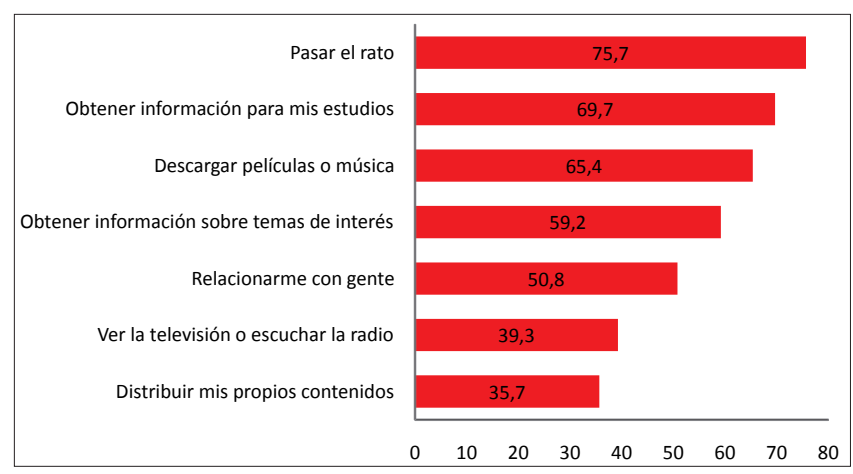

Figura 1. Usos de internet - “Uso internet para...” (\%) 
radio de lo que llaman "información sobre temas de interés" indica que no consideran esos medios como una fuente de información de primera necesidad, sino como formas de entretenimiento tradicional. De hecho, los grupos de discusión revelan que los contenidos consumidos en esos medios son la ficción (televisión) y la música (radio). Por otro lado, lo que los jóvenes consideran "información sobre temas de interés" es información sobre videojuegos, viajes, moda, deportes, sexualidad, salud y aspecto físico, y contenidos informativos sobre los artistas y personajes del mundo de la música, el cine y la televisión.

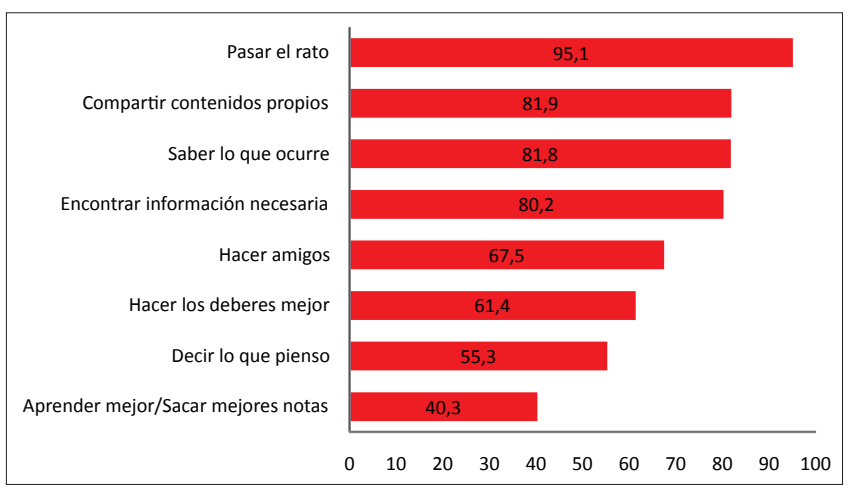

Figura 2. Percepción sobre la utilidad de internet - "Internet me posibilita..." (\%)

La figura 2 muestra la percepción de los jóvenes sobre las posibilidades que ofrece internet. La comparación entre los usos efectivos y esta percepción revela algunas singularidades en su forma de integrar la Red en su vida cotidiana. Si bien el entretenimiento y la información siguen siendo dos de las funciones principales atribuidas, también lo es la participación ("internet me posibilita compartir contenidos propios"). Sin embargo, la diferencia entre el uso efectivo para "compartir" y la percepción del potencial que ofrece para hacerlo sugiere que internet es vista como una extensión online de la vida offline, en tanto esos contenidos compartidos son, según afirman los jóvenes en los grupos de discusión, fotos y videos que se ponen a disposición de amigos que no están fuera de los círculos sociales de su vida offline.

\subsection{Criterios de evaluación de la información.}

En los grupos de discusión los jóvenes afirman que el rigor es esencial cuando se usa internet para obtener información útil para los estudios, pero que esto no resulta tan crítico en otros ámbitos, como el del entretenimiento. Surge por tanto la cuestión de los criterios para valorar la veracidad y/o utilidad de la información que obtienen en la Red. A veces reconocen que les faltan competencias:

- chica, bachillerato, Tarifa: "un trabajo de inglés, francés, lo que sea, que tienes que buscar cosas... muchas veces es tanta información que tú no sabes seleccionar bien cuál es realmente la que te vale la pena".

Se pueden señalar tres criterios principales para establecer la confianza en la información que se obtiene: por un lado, el sentido común, que podríamos encuadrar dentro de las prácticas de aprendizaje no formal, autodidacta:
- chica, ESO, Barcelona: “comparas con diferentes páginas y el sentido común que tú tengas. Leerlo y mirar si te parece verdad o no".

Otro criterio es el prestigio de la fuente, lo cual en principio parecería contradictorio con una orientación a la cultura participativa, propia del uso de la Red, en la que el prestigio no está vinculado a las instituciones formales:

- chico, bachillerato, Barcelona: "pero hay información de confianza, por ejemplo, está la Enciclopèdia Catalana, cada vez más amplia...";

- chica, bachillerato, Barcelona: "y la Encarta online. Pero $100 \%$ de fiabilidad no creo...".

Finalmente, un tercer criterio es la reiteración, es decir, la búsqueda de la misma información en diferentes lugares y la comprobación de que se mantiene consistente en las distintas fuentes:

- chica, bachillerato, Santiago de Compostela: "tienes que comprobar mucho para que te valga lo que estás haciendo, no te vale una página, tienes que mirar cinco o seis para hacer un trabajo".

\subsection{Información y sociabilidad.}

Los dos servicios orientados a la sociabilidad más utilizados por los jóvenes (las redes sociales y la mensajería instantánea) ofrecen posibilidad de análisis de sus mecanismos de valoración de la información. En este sentido, destaca que obtener información sobre temas de interés está en una modesta octava posición entre los usos de redes sociales (figura 3). Esta afirmación debe matizarse a partir de los comentarios de los propios jóvenes cuando son preguntados en profundidad en los grupos de discusión, dado que una de las funciones principales atribuidas a las redes sociales es pasar el rato comentando en grupo la información que encuentran en los perfiles de sus contactos. Saber qué hacen los amigos y amigas es, precisamente, la información útil para la vida cotidiana que los jóvenes consumen en las redes sociales que utilizan -Tuenti (68,5\%) y Facebook $(10,1 \%)$.

Las conversaciones rutinarias y las muletillas del lenguaje no están bien consideradas en la comunicación mediante mensajería instantánea

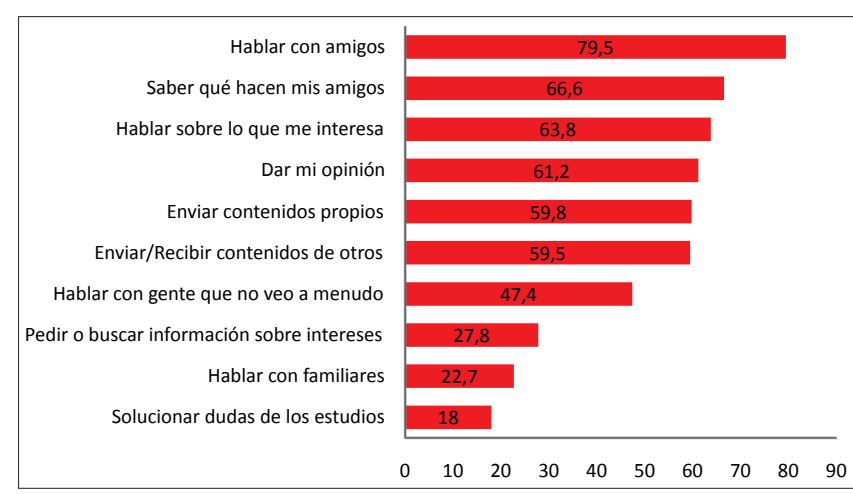

Figura 3. Usos de redes sociales (\%) 
Los criterios de evaluación de la información por parte de los jóvenes en los servicios de mensajería instantánea (principalmente Messenger) se formulan en términos de lenguaje. Los jóvenes manifiestan ciertas preferencias en la comunicación a través de Messenger. Muy valorada por ejemplo es la concisión, el "ir al grano". Las conversaciones rutinarias o las muletillas tan usadas en la comunicación verbal interpersonal no están bien consideradas. Un "hola" es bien recibido, pero una conversación llena de muletillas propias de la función fática (de comprobación o sin contenido) del lenguaje les resulta completamente falta de interés.

- Chica, bachillerato, Barcelona: "me parece que en Messenger mucha gente se ha creado una forma de conversación rutinaria, que es: 'Hola. Hola. ¿Qué tal? Bien, ¿y tú? También, ¿qué me explicas? Nada, ¿̇y tú? Tampoco'... Hay conversaciones que claramente no van a ninguna parte. Si empezamos con 'Buenas', vamos mal";

- chico, ESO, Tarifa: "hola, qué tal, qué te cuentas... Para eso no hablo".

- chico 2, ESO, Tarifa: "Uf... odio eso".

En los grupos de discusión con jóvenes se observa que estos otorgan una gran importancia a los intercambios de frases que cumplen una evidente función referencial (es decir, que contienen información relevante) o una función expresiva (iconos o frases que reflejan determinados estados de ánimo), según la ya clásica distinción de las funciones del lenguaje de Jakobson. Aunque estas afirmaciones se producen fundamentalmente entre los de mayor edad, cuyo uso es más reflexivo, los jóvenes en general son plenamente conscientes de que el canal está abierto por el simple hecho de estar presentes y conectados. Que alguien manifieste que está disponible es suficiente para entablar una conversación, que siempre debe estar orientada a "explicar algo interesante". Es posible de ese modo afirmar que los jóvenes han creado un código propio a partir de una competencia tecnológica y un capital cultural adquirido de forma autodidacta.

Los jóvenes han creado un código propio a partir de una competencia tecnológica y un capital cultural adquirido de forma autodidacta

\section{Conclusiones}

Los resultados discutidos en el presente artículo son consistentes si se comparan con las conclusiones de otras investigaciones españolas e internacionales (Winocur, 2006; Instituto Nacional de Tecnologías de la Comunicación, 2009; Ito et al., 2009; Bringué; Sádaba, 2008, 2009), y permiten extraer conclusiones específicas sobre los usos y las percepciones de la información en internet.

La comparación entre los datos obtenidos mediante encuesta y los matices aportados por los jóvenes en los grupos de discusión, describen un panorama en el que las motivaciones para conectarse y explorar la versatilidad técnica de internet como fuente de información son pasar el rato y buscar y compartir contenidos orientados a la sociabilidad y a sus intereses cotidianos, que incluyen, como no podría ser de otro modo, sus deberes escolares. Los jóvenes son conscientes de que internet les posibilita compartir contenidos generados por ellos mismos, lo cual hacen con el objetivo de estrechar lazos con sus amigos. El impulso participativo de los jóvenes en internet es consecuencia por tanto de su necesidad de sociabilidad.

Cabe destacar que los jóvenes separan los dos contextos fundamentales de su cotidianidad -el ocio y la educación formal- valorando de forma diferente la información que usan en cada uno de ellos. En el caso de la educación formal, el uso que hacen de internet ilustra formas características de búsqueda, valoración y utilización de la información, basadas en el sentido común, el prestigio de la fuente y la repetición. En su uso de la información orientada al ocio y la sociabilidad, los jóvenes se dotan de protocolos y códigos propios, construidos sobre una competencia tecnológica obtenida a partir de un proceso de aprendizaje no formal.

\section{Nota}

La investigación forma parte del proyecto financiado por el Ministerio de Industria, Turismo y Comercio (Plan Avanza) y coordinado por el Centre d'Estudis de la Fundació Catalana de l'Esplai (referencia TSI-040400-2008-42) que lleva por título Transformemos el ocio digital: un proyecto de socialización en el tiempo libre.

\section{Bibliografía citada}

Aranda, Daniel; Sánchez-Navarro, Jordi; Tabernero, Carlos. Jóvenes y ocio digital. Informe sobre el uso de herramientas digitales por parte de adolescentes en España. Barcelona: Editorial UOC, 2009.

http://in3.uoc.edu/opencms_portalin3/opencms/PDF/ jovenes_ocio_digital_250310.pdf

Bernete, Francisco. "Usos de las TIC, relaciones sociales y cambios en la socialización de las y los jóvenes". Revista de estudios de juventud, 2010, n. 88, pp. 97-114.

http://www.injuve.migualdad.es/injuve/contenidos. downloadatt.action?id=299824229

Bringué, Xavier; Sádaba, Charo. La generación interactiva en España. Barcelona: Ariel, 2009.

http://www.generacionesinteractivas.org/wp-content/ uploads/2009/12/La-Generación-Interactiva-en-España.pdf

Bringué, Xavier; Sádaba, Charo. La generación interactiva en Iberoamérica. Barcelona: Ariel, 2008.

http://www.scribd.com/doc/9305291/GeneracionesInteractivas-en-Iberoamerica-Ninos-y-adolescentes-antelas-pantallas

Buckingham, David. Más allá de la tecnología. Aprendizaje infantil en la era de la cultura digital. Buenos Aires: Manantial, 2008.

De-Certeau, Michel. The practice of everyday life. BerkeleyLos Angeles: University of California Press, 1984.

Dickinson, Roger; Murcott, Anne; Eldridge, Jane; Leader, Simon. "Breakfast, time, and 'Breakfast time': television, 
food, and the organization of consumption". Television and new media, 2001, v. 2, n. 3, pp. 235-256.

Espinar-Ruiz, Eva; González-Río, María-José. “Jóvenes en las redes sociales virtuales: un análisis exploratorio de las diferencias de género". Feminismo/s: Revista del Centro de Estudios sobre la Mujer de la Universidad de Alicante, 2009, n. 14, pp. 87-106.

http://rua.ua.es/dspace/bitstream/10045/13302/1/Femi nismos_14_06.pdf

Gee, James-Paul. Situated language and learning: a critique of tradicional schooling. New York: Routledge, 2004.

Geertz, Clifford. La interpretación de las culturas. Barcelona: Gedisa, 1995.

Goffman, Erving. La presentación de la persona en la vida cotidiana. Buenos Aires: Amorrortu, 1994.

Gordo-López, Ángel J. (coord). Jóvenes y cultura Messenger: Tecnología de la información y la comunicación en la sociedad interactiva. Madrid: Instituto de la Juventud, 2006.

Grossberg, Lawrence. "El corazón de los estudios culturales: contextualidad, construccionismo y complejidad". Tabula rasa, 2009, n. 10, pp. 13-48.

Hagen, Ingunn. "'We can't just sit the whole day watching TV': negotiations concerning media use among youngsters and their parents". Young, 2007, v. 15, n. 4, pp. 369-393.

Highmore, Ben. Everyday life and cultural theory: an introduction. Londres: Routledge, 2001.

Instituto Nacional de Tecnologías de la Comunicación. Estudio sobre hábitos seguros en el uso de las TIC por parte de niños y adolescentes y e-confianza de los padres. Madrid: Inteco, 2009.

http://www.inteco.es/file/O4-7XOFfwOb7HFjdHHpx7Q

Ito, Mizuko; Baumer, Sonja; Bittanti, Matteo; Boyd, Danna; Cody, Rachel; Herr-Stephenson, Becky; Horst, Heather A.; Lange, Patricia G.; Mahendran, Dilan; Martinez, Katynka; Pascoe, C. J.; Perkel, Dan; Robinson, Laura; Sims, Christo; Tripp, Lisa. Hanging out, messing around, and geeking out: kids living and learning with new media. Cambridge, MA: MIT Press, 2009.

Jenkins, Henry; Purushotma, Ravi; Clinton, Katherine; Weigel, Margaret; Robison, Alice J. Confronting the challenges of participatory culture: media education for the $21^{\text {st }}$ century. Chicago: The MacArthur Foundation, 2008.

http://newmedialiteracies.org/files/working/NMLWhite Paper.pdf

Livingstone, Sonia. “Children's use of the internet: Reflec- tions on the emerging research agenda". New media and society, 2003, v. 5, n. 2, pp. 147-166.

http://eprints.Ise.ac.uk/415/1/NMS-use-of-internet.pdf

Livingstone, Sonia. "Taking risky opportunities in youthful content creation: teenagers' use of social networking sites for intimacy, privacy and self-expression". New media and society, 2008 , v. 10 , n. 3, pp. 393-411.

Livingstone, Sonia. "The challenge of engaging youth online". European journal of communication, 2007, v. 22, n. 2, pp. 165-184.

Livingstone, Sonia; Helsper, Ellen. "Gradations in digital inclusion: children, young people and the digital divide". New media and society, 2007, v. 9, n. 4, pp. 671-696.

http://eprints.Ise.ac.uk/2768/1/Gradations_in_digital_in clusion_(LSERO).pdf

Lull, James. Medios, comunicación y cultura. Aproximación global. Buenos Aires: Amorrortu, 1997.

McMillan, Sally J.; Morrison, Margaret. "Coming of age with the internet". New media and society, 2006, v. 8, n. 1, pp. 73-95.

Orozco, Guillermo. Televisión y audiencias: un enfoque cualitativo. Madrid: Ediciones de la Torre, 1996.

Prensky, Marc. "Digital natives, digital immigrants". On the horizon, 2001, v. 9, n. 5, pp. 1-6.

http://www.marcprensky.com/writing/Prensky\%20-\%20Dig ital\%20Natives,\%20Digital\%20Immigrants\%20-\%20Part1.pdf

Rheingold, Howard. Smart mobs. The next social revolution. Cambridge, MA: Perseus Publishing, 2002.

Rubio-Gil, Ángeles. Adolescentes y jóvenes en red. Madrid, Instituto de la Juventud, 2009.

Rubio-Gil, Ángeles. "Generación digital: patrones de consumo de internet, cultura juvenil y cambio social". Revista de estudios de juventud, 2010, n. 88, pp. 201-221.

http://www.injuve.migualdad.es/injuve/contenidos. downloadatt.action?id $=290289448$

Selwyn, Neil. "Doing IT for the kids: re-examining children, computers and the "information society'". Media, culture and society, 2003, v. 25, n. 3, pp. 351-378.

Storey, John. Cultural consumption and everyday life. Nueva York: Hodder Arnold, 1999.

Winocur, Rosalía. "Internet en la vida cotidiana de los jóvenes". Revista mexicana de sociología, 2006, v. 68, n. 3, pp. 551-580.

http://www.ejournal.unam. $m x / r m s / 2006-3 / R M S 006000$ 305.pdf

\section{Suscripción EPI sólo online}

Pensando sobre todo en los posibles suscriptores latinoamericanos, ya no es obligatorio pagar la suscripción impresa de EPI para acceder a la online.

EPI se ofrece a instituciones en suscripción "sólo online" a un precio considerablemente más reducido (90 euros/año), puesto que en esta modalidad no hay que cubrir los gastos de imprenta ni de correo postal. 